\title{
Avaliação da Energia de Soldagem e da Adição de Arame Frio nas Características Dimensionais, Químicas e Metalúrgicas do Metal de Solda Obtido por Arco Submerso em Aços Inoxidáveis Lean Duplex UNS S32304
}

\author{
Ronaldo Cardoso Junior ${ }^{1}$, Indiana Oliveira², Danielle Silva Mendes², Luiza Esteves ${ }^{3}$, Neice Ferreira dos Santos ${ }^{3}$, \\ Paulo José Modenesi² \\ ${ }^{1}$ ESAB Industria e Comércio LTDA, Gerencia de Produtos, Contagem, MG, Brasil. \\ ${ }^{2}$ Universidade Federal de Minas Gerais - UFMG, Departamento de Engenharia Metalúrgica, Belo Horizonte, MG, Brasil. \\ ${ }^{3}$ Centro de Desenvolvimento da Tecnologia Nuclear - CDTN, Belo Horizonte, MG, Brasil.
}

Recebido: 11 Jan., 2018

Aceito: 03 Jun., 2018

E-mail: ronaldo.junior@esab.com.br $(\mathrm{RCJ})$
Resumo: A energia de soldagem (HI) máxima indicada para soldagem dos aços inoxidáveis duplex de $2,5 \mathrm{~kJ} . \mathrm{mm}^{-1}$ representa um fator limitante para o aumento de produtividade na soldagem dos mesmos, sendo a adição de arame frio uma alternativa interessante à essa questão. Avaliou-se a influência da energia de soldagem e da adição do arame frio nas características dimensionais, químicas e metalúrgicas do metal de solda (MS) obtido por arco submerso (SAW) em aço "lean" duplex UNS S32304, utilizando-se o consumível ER2209. Empregou-se o sistema ICE ${ }^{\mathrm{TM}}$ que adiciona um arame frio entre dois arames energizados "twin" para executar cordões sobre chapas, empregando-se quatro diferentes $\mathrm{HI}$ e variando-se a porcentagem de arame frio em relação aos arames quentes em 0, 50 e 100\%. Foram realizadas análise química, macrografia, análise dimensional, microscopia ótica e MEV/EDS do MS. A microestrutura do MS foi constituída de uma matriz ferrítica com a presença de austenita de contorno de grão, de Widmanstatten e intragranular e inclusões não metálicas, não sendo observado a ocorrência de precipitados deletérios como $\sigma, \chi$ e nitreto de cromo. A adição de arame frio aumentou a taxa de deposição e provocou a redução da diluição, produzindo soldas com maiores números equivalentes de resistência à corrosão por pite, principalmente para maiores $\mathrm{HI}$, e maiores frações de austenita. Isso indica que o aumento da velocidade de alimentação de arame frio propicia o aumento da produtividade e da resistência à corrosão por pite do MS.

Palavras-chave: Lean duplex; Arame frio; SAW; Diluição.

\section{Evaluation of Heat Input and Cold Wire Addition on Dimensional, Chemical and Metallurgical Characteristics of The Weld Metal Obtained by Submerged Arc Welding in UNS S32304 Lean Duplex Steel}

\begin{abstract}
The maximum recommended welding energy $(\mathrm{HI})$ of $2.5 \mathrm{~kJ} \cdot \mathrm{mm}^{-1}$ represents a limiting factor for the productivity increase for duplex stainless steels welding, and the addition of cold wire is an interesting alternative to this question. It has been evaluated the influence of welding energy and the addition of cold wire on the dimensional, chemical and metallurgical characteristics of the weld metal (WM) of SAW welds in lean duplex steel UNS S32304, using the ER2209 filler metal. The ICE ${ }^{\text {TM }}$ system was used to add a cold wire between two twin energized wires to produce bead-on-plate welds, applying four different HIs and varying the percentage of cold wire (AF) in relation to the hot wires at 0, 50 and 100\%. It has been performed chemical analysis, macrography, dimensional analysis, optical microscopy and MEV / EDS in the WM of all conditions. The WM microstructure was constituted by a ferritic matrix with grain boundary, Widmanstatten and intergranular austenite and non-metallic inclusions. No deleterious precipitates such as $\sigma, \chi$ and chromium nitride were observed. The addition of cold wire increased the deposition rate and reduced dilution, achieving higher pitting resistance equivalent number, mainly for higher $\mathrm{HI}$ conditions, and higher austenite fractions. This indicates that the higher cold wire feed speed provides increased productivity and WM pitting corrosion resistance.
\end{abstract}

Key-words: "Lean" duplex, Cold wire, SAW, Dilution.

\section{Introdução}

Com o aumento da exploração de petróleo no Brasil e no mundo, vem aumentando de forma significativa a necessidade de materiais soldáveis com maior resistência mecânica e resistência à corrosão. Buscando atender este quesito, os aços inoxidáveis duplex (AID) 
Avaliação da Energia de Soldagem e da Adição de Arame Frio nas Características Dimensionais, Químicas e Metalúrgicas do Metal de Solda Obtido por Arco Submerso em Aços Inoxidáveis Lean Duplex UNS S32304

vêm se apresentando como uma excelente opção [1]. Os AID podem ser definidos como aqueles que apresentam uma microestrutura mista de ferrita e austenita, na proporção de aproximadamente $50 \%$ para ambas fases [2]. Esses materiais foram desenvolvidos há mais de 70 anos [3] e tem seu uso comercial iniciado de forma extensiva na década de 70 [4]. Os principais elementos de liga utilizados nessa classe de materiais são cromo e níquel, porém frequentemente são adicionados em menores proporções elementos como molibdênio, nitrogênio, cobre, silício e tungstênio [5].

Os AID têm muito em comum com aços inoxidáveis austeníticos e ferríticos, mas possuem várias propriedades únicas. Eles têm melhor resistência à corrosão sob tensão do que a maioria das ligas austeníticas, maior dureza do que a maioria das ligas ferríticas e maior resistência mecânica do que a maioria de ambos [2]. A ferrita promove maior resistência mecânica e melhor resistência à corrosão sob tensão (SCC - "stress corrosion crack") [6], enquanto a austenita favorece a boa resistência à corrosão generalizada e ductilidade. Uma excelente resistência à corrosão localizada em meios neutros e ácidos contendo cloretos pode ser obtida devido aos elevados teores de $\mathrm{Cr}$, Mo e N [6]. De acordo com Westin [7], em geral, a maior resistência mecânica dos AID também contribui para uma maior resistência à fadiga e ao desgaste por abrasão. Os AID podem ser uma alternativa economicamente viável em relação às ligas austeníticas de equivalente resistência à corrosão, especialmente em aplicações em que o dobro de limite de escoamento pode ser levado em consideração no projeto para redução da espessura de parede dos componentes [7]. Apesar das excelentes propriedades mecânicas e de resistência à corrosão, os AID 2205 ainda apresentam um elevado custo, principalmente devido aos altos teores de Ni e Mo. Desta maneira, a busca por ligas com microestrutura mista, porém com menor custo levou ao desenvolvimento dos aços inoxidáveis "lean" duplex (AILD).

Nos AILD, o níquel é parcialmente substituído por elementos formadores de austenita, como o manganês e nitrogênio, e o teor de molibdênio é reduzido. Eles apresentam como composição química típica 20-24\% de cromo, 1-5\% de níquel, 0,1-0,3\% de molibdênio e 0,10-0,22\% de nitrogênio [7]. O "lean" duplex comercialmente mais comum é a liga UNS $\$ 32304$ que apresenta composição nominal 23\% de cromo, $4 \%$ de níquel, adições de molibdênio de até $0,6 \%$, nitrogênio e manganês, o qual possui o PREN ("Pitting resistance equivalent number") entre 24 e 25 , similar à liga austenítica AISI 316L, porém com o dobro do limite de escoamento e melhor resistência à corrosão sob tensão [7] devido à maior proporção de ferrita. O PREN é o parâmetro geralmente aceito como uma estimativa da resistência à corrosão localizada dos aços inoxidáveis e pode ser definido pela Equação 1 [8]. O AILD UNS S32304 vem sendo utilizado em tanques de estocagem na indústria de papel e celulose, pontes, estações de tratamento de água, mineração e na indústria offshore [7]. Sua soldabilidade geralmente é boa quando consumíveis com maiores teores de níquel ("over-alloyed") são utilizados [6], entretanto para uma dada composição química do metal de base, a microestrutura da zona termicamente afetada (ZTA) continua dependendo exclusivamente do ciclo térmico [1].

$$
\text { PREN }=1 .(\% \mathrm{Cr})+3,3 .(\% \mathrm{Mo})+16 \cdot(\% \mathrm{~N})
$$

Em função da complexidade química dos AID, um grande número de reações de precipitação, dependentes do tempo e temperatura, podem ocorrer quando o material é submetido a elevadas temperaturas, a uma faixa de temperatura abaixo de $1000^{\circ} \mathrm{C}$. Muitos dos precipitados formados fragilizam a microestrutura dos AID, entre estes podem ser citados a fase sigma $(\sigma)$, chi $(\chi)$, alfa prime $\left(\alpha^{\prime}\right)$ e o nitreto de cromo. A adição ou aumento do teor de $\mathrm{Cr}$, Mo e $\mathrm{W}$ tende a acelerar a formação dos mesmos, principalmente da $\sigma$ e $\chi[9]$.

Quando os AID são soldados, o ciclo térmico imposto ao material pode prejudicar significativamente suas propriedades, especialmente a tenacidade e resistência à corrosão localizada [10]. Como os AID possuem microestrutura $100 \%$ ferrítica a altas temperaturas, elevadas velocidades de resfriamento podem provocar a ferritização excessiva da zona afetada (ZTA) e do metal de solda (MS) [11,12], bem como a formação de nitretos de cromo [9,13], enquanto o resfriamento lento ou repetidos ciclos térmicos entre $600{ }^{\circ} \mathrm{C}$ e $1000{ }^{\circ} \mathrm{C}$ favorecerá a formação de fases intermetálicas, especialmente nos AID com maiores adições de elementos de liga [13]. A ferritização do MS geralmente não é um problema quando um consumível com maior teor de Ni é empregado. Na prática, a formação de fases intermetálicas é raramente um problema na soldagem para os AID standard e "lean" duplex, entretanto um restrito controle do procedimento de soldagem é requerido para os super e hiper duplex [13].

A energia de soldagem (HI) desempenhará um papel importante na história térmica da junta soldada e na formação da estrutura da junta [14]. O impacto do emprego de uma HI muito alta nos AID é o favorecimento da 
formação de fases intermetálicas deletérias e o excessivo crescimento de grão ferrítico, enquanto uma energia muito baixa favorecerá uma excessiva ferritização. Neste contexto, se recomenda uma $\mathrm{HI}$ na faixa de 0,5 a 2,5 kJ.mm ${ }^{-1}$ para soldagem do AILD UNS S32304 [10,13]. O valor superior restringe o uso de maiores velocidades de alimentação de arame (WFS) e corrente de soldagem, o que representa um fator limitante para aumentar a produtividade ao soldar os AID, especialmente na soldagem por arco submerso (SAW) no qual um alto nível de energia de soldagem é geralmente aplicado. A adição de arame frio representa uma alternativa interessante, pois permite o aumento da taxa de deposição sem o aumento de HI [14-16]. Algumas pesquisas foram relatadas sobre a adição de arame frio em SAW para ligas de aço carbono [14-17], mas nenhuma foi encontrada para AID.

Um dos sistemas de adição de arame frio no processo SAW comercialmente disponível no mercado é o ICE ${ }^{\mathrm{TM}}$, que consiste basicamente de um sistema "twin" (arames geminados) com adição de um arame frio entre os arames "twin" energizados, como ilustrado na Figura 1. Portanto, no processo SAW-ICE três arames são adicionados na mesma poça de fusão, sendo dois arames geminados quentes ("twin") e um frio equidistante aos arames quentes. Pelo conceito de construção do equipamento, a velocidade de alimentação do arame frio é parametrizada como uma relação, em porcentagem, da velocidade de alimentação dos arames energizados. Nesse trabalho, essa porcentagem será tratada como AF (arame frio).

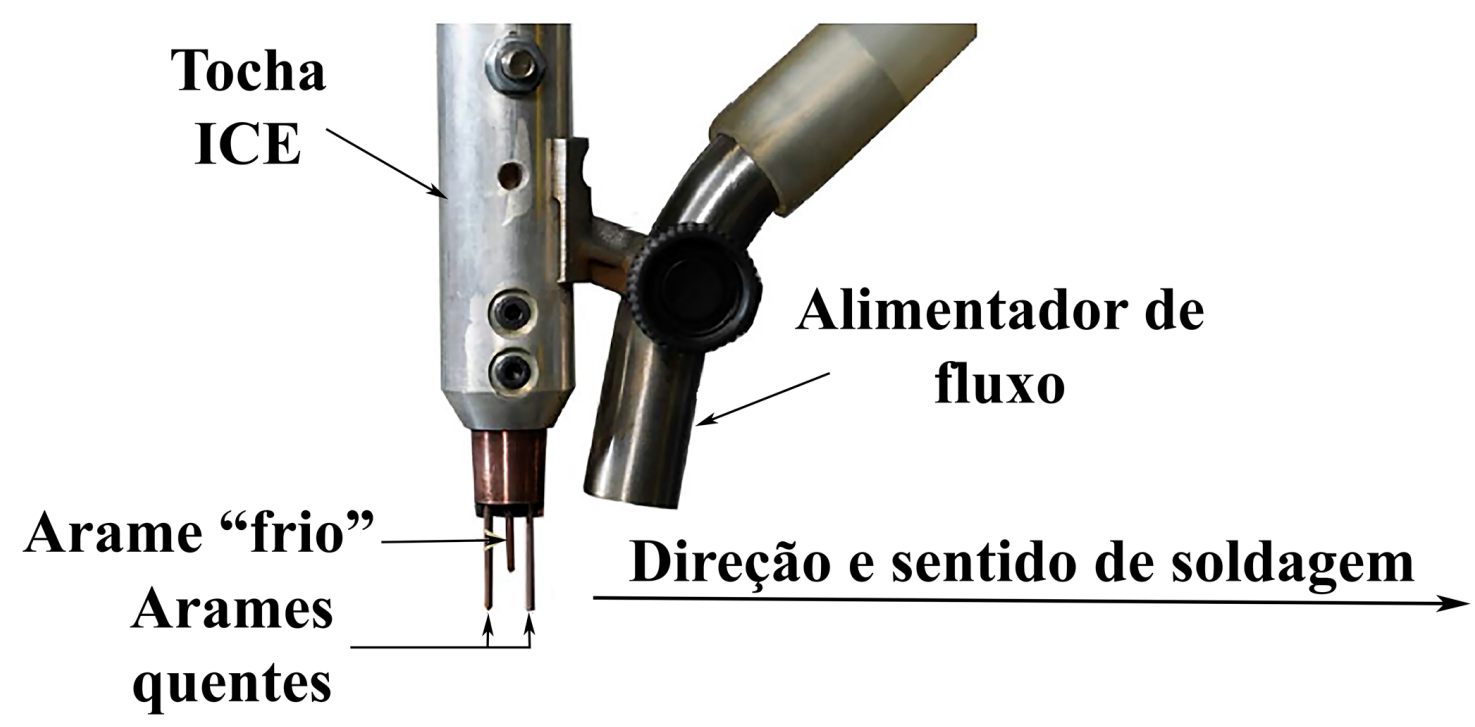

Figura 1. Alimentação de arame do sistema ICE ${ }^{\mathrm{TM}}$ [17].

Portanto, o objetivo desse trabalho é avaliar a influência da energia de soldagem e da velocidade de adição do arame frio nas características químicas e metalúrgicas do metal de solda SAW em AILD UNS S32304, utilizando-se consumível (arame) de classificação ASME II Part C ER2209 [18].

\section{Materiais e Métodos}

Foram obtidas 12 condições de soldagem, variando-se a adição de arame frio e a energia de soldagem. Para tal avaliação, foram realizados cordões sobre chapas ("bead-on-plate"), utilizando-se o processo SAW com adição

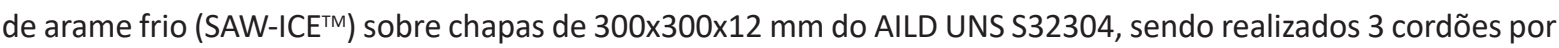
chapa, preservado espaçamento de $75 \mathrm{~mm}$ entre os cordões e as bordas e aproximadamente $20 \mathrm{~mm}$ nas bordas para início e término do cordão.

Para todos os experimentos empregou-se uma mesma combinação de consumíveis (fluxo e arame) composta pelo arame sólido de especificação ASME II Part C A5.9 ER2209 [18], de diâmetro de 2,50 mm, e pelo fluxo OK Flux 10.93. Segundo informação fornecida em catálogo pelo fabricante, este é um fluxo aglomerado básico não ligado para soldagem ao arco submerso de aços inoxidáveis de classificação EN ISO 14174 S A AF 25654 DC, 
com índice de basicidade nominal de 1,70, densidade de $1 \mathrm{~kg} / \mathrm{dm}^{3}$ e escória do tipo $\mathrm{CaF}_{2}-\mathrm{Al}_{2} \mathrm{O}_{3}-\mathrm{SiO}_{2}$. A Tabela 1 apresenta a composição química dos materiais utilizados nesse trabalho no estado "como recebido". Esses valores foram obtidos utilizando-se as mesmas técnicas experimentais empregadas para o MS, descritas posteriormente nesta secção.

Tabela 1. Composição química, em \% de massa, dos materiais utilizados e suas respectivas faixas normativas.

\begin{tabular}{cccccccccccc}
\hline \multirow{2}{*}{ Material } & Resultado & \multicolumn{10}{c}{ Concentração dos elementos em peso (\%) } \\
\cline { 3 - 12 } & & $\mathbf{C}$ & Mn & Si & Cr & Ni & P & S & Cu & Mo & N \\
\multirow{2}{*}{ Chapa } & UNS S323041 & 0,030 & 2,00 & 1,00 & $22,0-24,0$ & $3,5-5,5$ & 0,035 & 0,015 & $0,10-0,60$ & $0,1-0,6$ & $0,05-0,20$ \\
& Real & 0,019 & 1,23 & 0,42 & 22,0 & 3,6 & 0,030 & 0,000 & 0,41 & 0,24 & 0,10 \\
Arame & ER22092 & 0,030 & $0,25-0,75$ & 0,90 & $21,5-23,5$ & $7,5-9,5$ & 0,030 & 0,030 & 0,75 & $2,5-3,5$ & $0,08-0,20$ \\
& Real & 0,012 & 1,70 & 0,47 & 22,8 & 8,7 & 0,020 & 0,001 & 0,10 & 3,10 & 0,17 \\
\hline
\end{tabular}

${ }^{1}$ de acordo com ASTM A240 / A240M-17, 2017 [19]. ${ }^{2}$ de acordo com ASME Section II-Materials Part C, 2017 [18].

A soldagem foi executada utilizando-se um cabeçote de soldagem SAW-ICE (SAW "Integrated Cold Electrode") e fonte de soldagem AC/DC de 1000 amperes, Figura 2.

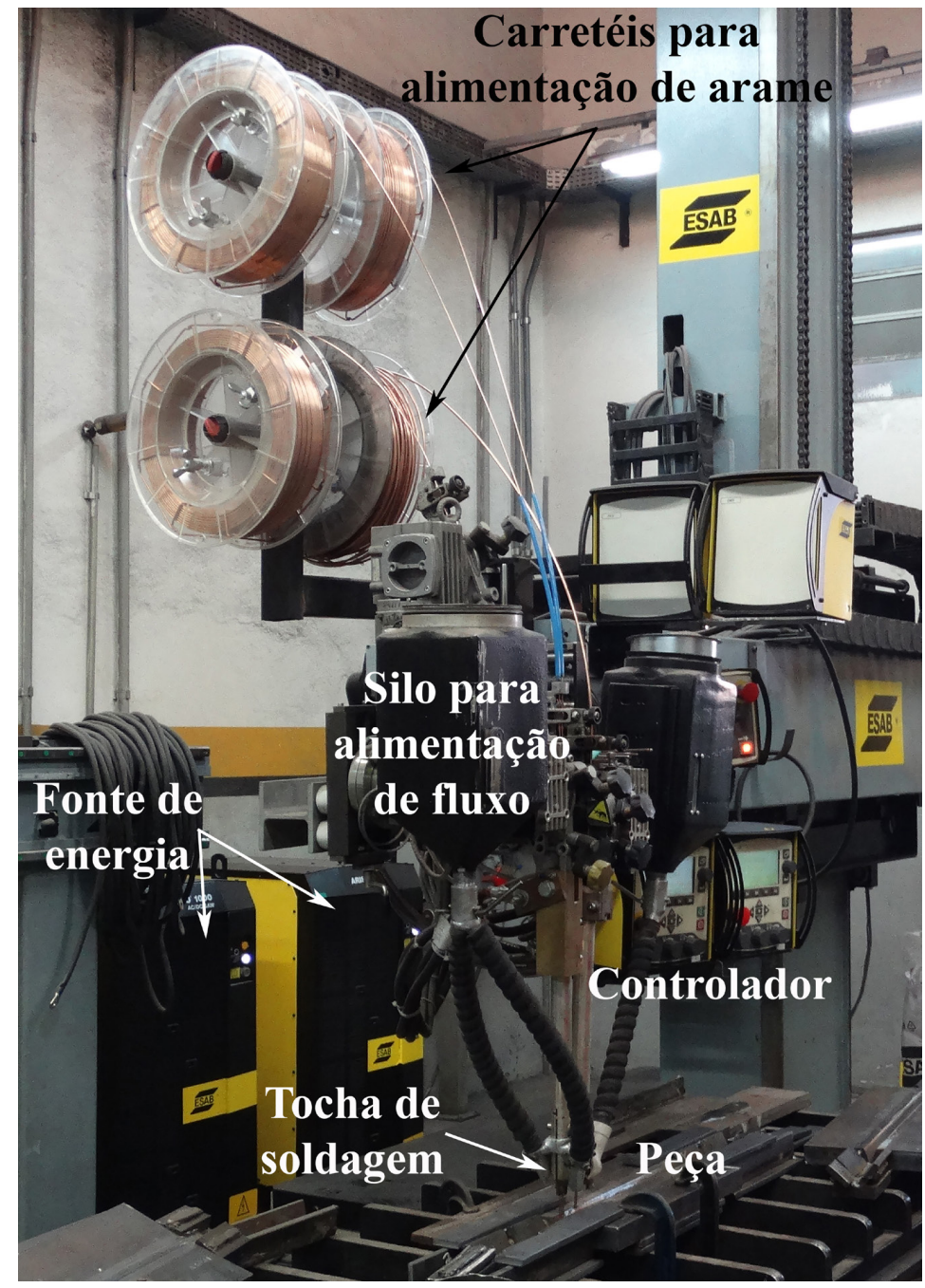

Figura 2. Foto do equipamento utilizado para soldagem dos corpos de prova. 
As chapas foram esmerilhadas e o fluxo de soldagem ressecado a $300^{\circ} \mathrm{C}$ por $2 \mathrm{~h}$, conforme recomendação do fabricante. Para todos os experimentos a corrente de soldagem, polaridade (DC+), tensão de soldagem e a distância entre a peça e o bico de contato ( $D P B C=30 \mathrm{~mm}$ ) foram mantidas constantes, variando-se apenas a velocidade de soldagem para se obter 4 distintas energias de soldagem. Para cada uma delas, variou-se a adição de arame frio (AF) em $0 \%, 50 \%$ e 100\%. Esses valores percentuais correspondem a proporção entre a velocidade de alimentação do arame frio em relação aos arames quentes. Dessa forma, foram obtidas 12 diferentes condições de soldagem, conforme detalhado na Tabela 2. Ressalta-se que a energia de soldagem de $2,7 \mathrm{~kJ} . \mathrm{mm}^{-1}$, que está acima do limite recomendado pela literatura, foi empregada propositalmente com o objetivo de estudar o impacto da mesma em uma condição em que há a adição de arame frio, o que provoca um efeito refrigerante na poça de fusão e deve afetar o resfriamento da junta soldada.

Tabela 2. Parâmetros de soldagem utilizados nos experimentos.

\begin{tabular}{|c|c|c|c|c|c|c|}
\hline Teste & AF (\%) & $\begin{array}{l}\text { Vel. de alimentação } \\
\text { dos arames quentes } \\
\left(\mathrm{m} \cdot \mathrm{min}^{-1}\right)\end{array}$ & $\begin{array}{l}\text { Corrente de } \\
\text { Soldagem (A) }\end{array}$ & $\begin{array}{c}\text { Tensão de } \\
\text { Soldagem (V) }\end{array}$ & $\begin{array}{l}\text { Vel. Soldagem } \\
\left.\text { (cm.min }{ }^{-1}\right)\end{array}$ & $\mathrm{HI}\left(\mathrm{kJ} \cdot \mathrm{mm}^{-1}\right)$ \\
\hline $1,6-0$ & 0 & 2,3 & 550 & 37 & 75 & 1,6 \\
\hline $1,6-50$ & 50 & 2,1 & & & & \\
\hline $1,6-100$ & 100 & 2,1 & & & & \\
\hline $1,9-0$ & 0 & 2,3 & 550 & 37 & 65 & 1,9 \\
\hline $1,9-50$ & 50 & 2,1 & & & & \\
\hline $1,9-100$ & 100 & 2,1 & & & & \\
\hline $2,2-0$ & 0 & 2,3 & 550 & 37 & 55 & 2,2 \\
\hline $2,2-50$ & 50 & 2,1 & & & & \\
\hline $2,2-100$ & 100 & 2,1 & & & & \\
\hline $2,7-0$ & 0 & 2,3 & 550 & 37 & 45 & 2,7 \\
\hline $2,7-50$ & 50 & 2,1 & & & & \\
\hline $2,7-100$ & 100 & 2,1 & & & & \\
\hline
\end{tabular}

Na Tabela 2, as variáveis independentes são a corrente, tensão e velocidade de soldagem, enquanto as variáveis dependentes são a velocidade de alimentação dos arames quentes, que é uma resposta do equipamento de soldagem à corrente parametrizada, a velocidade de alimentação do arame frio, que está relacionada à velocidade dos arames quentes e a energia de soldagem, que está relacionada com a corrente, tensão e velocidade de soldagem.

Para análise química do MS, foram utilizadas a absorção de infravermelho após fusão para os elementos C e S (equipamento LECO-CS844), a termocondutividade após fusão para o N (equipamento LECO-TC436DR) e espectroscopia de emissão ótica para os demais elementos químicos (equipamento Thermo ARL-4460). A análise foi realizada na superfície do cordão após desbaste por fresamento, respeitando-se a superfície da chapa como profundidade máxima de desbaste.

Para análise metalográfica da secção transversal, corpos de provas foram retirados na região central do cordão (no meio do comprimento). Para macrografia, as amostras foram lixadas até granulometria de 1000 mesh, atacadas com reagente Behara II [20] e fotografadas com câmera digital com lente para macrofotografia. A largura, penetração, reforço e as áreas depositada e fundida (Figura 3) foram medidos em pelo menos 3 regiões do cordão de solda. A taxa

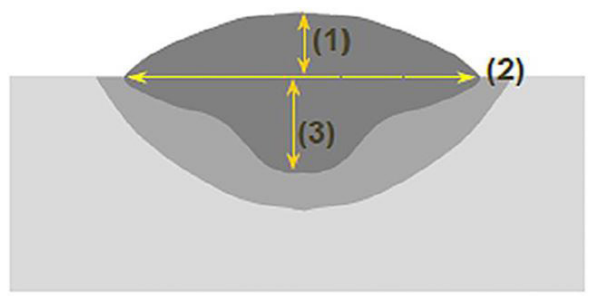

(a)

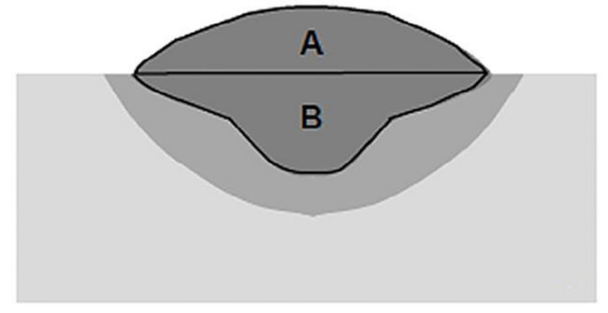

(b)

Figura 3. Perfil dimensional do cordão de solda. Sendo: (a) 1: reforço, 2: largura, 3: penetração (b) A: área depositada, B: área fundida [17]. 
de deposição (TD) foi calculada a partir da área depositada e da velocidade de soldagem. Como variou-se apenas a velocidade de soldagem, mantendo-se a corrente de soldagem e DPBC constantes para todos os testes, foi calculada a média das TD agrupando-se todos as condições de AF igual a 0, 50 e 100\%.

A partir de todas as medições dimensionais e de diluição, foi realizado o tratamento estatístico dos parâmetros. Os dados foram normalizados e em seguida foi realizada a regressão linear múltipla, utilizando-se os seguintes regressores: $\mathrm{HI}$; AF; $\mathrm{HI}^{2}$; $\mathrm{AF}^{2}$; HIXAF. O tratamento estatístico foi realizado com o software MINITAB. Para a construção dos modelos por regressão múltipla, as variáveis de entrada foram normalizadas por (Equação 2):

$$
X_{\text {Nor }}=(X-\text { Média }) / \text { Desvio Padrão }
$$

Os corpos de prova utilizados para microscopia ótica (MO) e eletrônica (MEV) foram embutidos a frio com resina epóxi e passaram por lixamento e posterior polimento com pasta de diamante nas granulometrias de 9, 6 e $3 \mu \mathrm{m}$, sendo atacados posteriormente com reagente Behara II. Foi utilizado microscópio ótico (Leica-2500M) e microscópio eletrônico de varredura (MEV) equipado com espectroscopia por Dispersão de Energia de Raios X (EDS) (FEI-Quanta FEG 250). O EDS foi empregado para estimar a composição química das inclusões não metálicas do MS.

Nas amostras de $\mathrm{MO}$ ainda não atacadas, foi determinado a fração volumétrica das inclusões não metálicas utilizando-se 10 campos aleatórios com aumento de 1000X. A fração das fases austenita e ferrita foi determinada utilizando-se 5 campos aleatórios, com ampliação de 500X, obtidos por MO em amostras atacadas.

\section{Resultados e Discussão}

A Figura 4 apresenta as macrografias das juntas soldadas das 12 condições de soldagem executadas nesse trabalho. Através da análise visual das mesmas, é possível observar soldas isentas de qualquer tipo de defeito macroscópico. Além disso, constata-se o aumento da área depositada em função da adição de arame frio, tal efeito

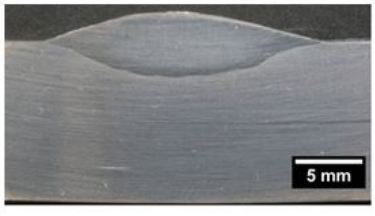

$1,6-0$

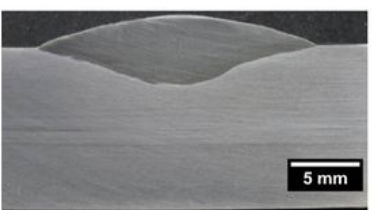

$1,9-0$

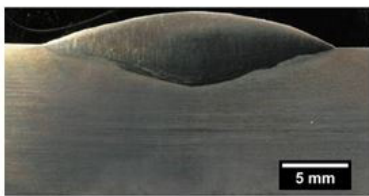

2,2-0

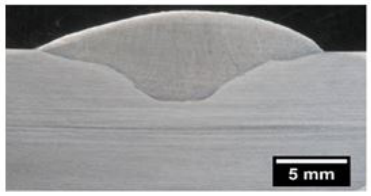

2,7-0

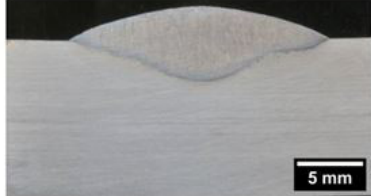

$1,6-50$

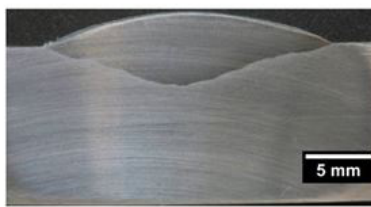

$1,9-50$

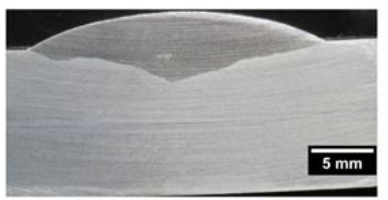

2,2-50

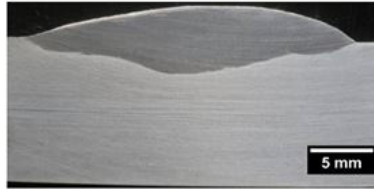

2,7-50

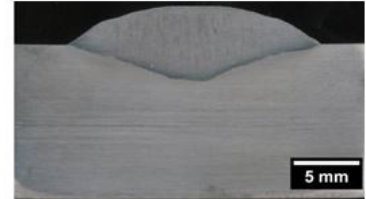

$1,6-100$

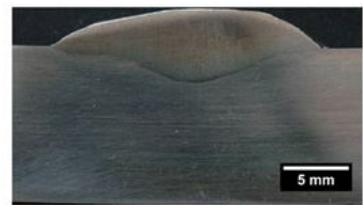

1,9-100

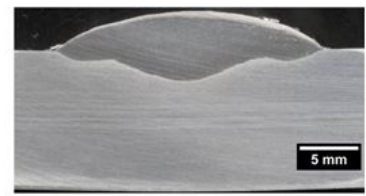

2.2-100

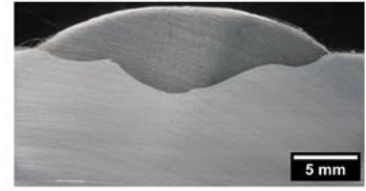

2,7-100

Figura 4. Macrografias das seções transversais dos cordões de solda. 
é mais notório visualmente quando se compara as condições extremas de 0 e $100 \%$ de AF para cada energia de soldagem. O impacto da maior área depositada é a maior taxa de deposição e possível menor diluição, fatores que serão detalhados adiante.

\subsection{Análise dimensional do cordão}

A Tabela 3 apresenta os parâmetros dimensionais dos cordões e a diluição para cada condição de soldagem. Para uma mesma $\mathrm{HI}$, a área depositada (AD) e a área total (At) aumentaram com o aumento de $\mathrm{AF}$, devido ao aumento da taxa de deposição provocada pela adição do arame frio e a manutenção da velocidade de soldagem constante, o que afetou a diluição. Os outros parâmetros não apresentaram uma tendência clara de variação, o que será reforçado pela análise estatística será apresentada em seguida.

Os resultados da regressão linear dos dados da Tabela 3 normalizados são apresentados na Tabela 4. Segundo Quinino et al. [21], um modelo é considerado adequado para o nível de significância de $5 \%$ quando o valor de $\mathrm{R}^{2}$ é superior a $79 \%$, considerando o número de regressores igual a $5(k=5)$ e o tamanho da amostra igual a $12(n=12)$, como é o caso da análise realizada. Assim, o modelamento pode ser considerado adequado para o reforço, área depositada, área fundida, área total e diluição, enquanto a largura e, principalmente, a penetração não apresentaram uma correlação clara com a energia de soldagem e adição de arame frio do ponto de vista estatístico.

A regressão linear indica que o reforço aumenta com a energia de soldagem e a adição de arame frio, sendo o efeito de $\mathrm{HI}$ mais forte que o de AF; a área depositada e área total aumentam com $\mathrm{HI}$ e AF, sendo a $\mathrm{HI}$ mais relevante que AF; a área fundida: aumenta com $\mathrm{HI}$ e reduz com AF; a diluição: reduz com $\mathrm{HI}$ com AF, e AF possui

Tabela 3. Parâmetros dimensionais médios e diluição média dos cordões de solda.

\begin{tabular}{lccccccc}
\hline Condição & $\mathbf{L}(\mathbf{m m})$ & $\mathbf{P}(\mathbf{m m})$ & $\mathbf{R}(\mathbf{m m})$ & Afund $\left(\mathbf{m m}^{2}\right)$ & $\mathbf{A D}\left(\mathbf{m m}^{2}\right)$ & $\mathbf{A t}\left(\mathbf{m m}^{2}\right)$ & Diluição (\%) \\
$1,6-0$ & 20,5 & 2,8 & 2,2 & 32,1 & 28,7 & 60,7 & $53 \%$ \\
$1,6-50$ & 19,8 & 3,1 & 2,5 & 32,6 & 34,2 & 66,8 & $49 \%$ \\
$1,6-100$ & 20,0 & 2,7 & 2,9 & 27,1 & 41,3 & 68,3 & $40 \%$ \\
$1,9-0$ & 20,7 & 2,9 & 2,4 & 31,2 & 31,7 & 62,8 & $50 \%$ \\
$1,9-50$ & 21,2 & 3,4 & 2,5 & 35,0 & 36,9 & 71,9 & $49 \%$ \\
$1,9-100$ & 20,2 & 2,7 & 2,9 & 28,2 & 45,3 & 73,6 & $38 \%$ \\
$2,2-0$ & 22,7 & 2,7 & 2,5 & 35,0 & 37,1 & 72,1 & $49 \%$ \\
$2,2-50$ & 24,8 & 3,0 & 2,9 & 36,8 & 48,3 & 85,1 & $43 \%$ \\
$2,2-100$ & 23,2 & 2,9 & 3,1 & 35,2 & 51,7 & 86,9 & $40 \%$ \\
$2,7-0$ & 21,2 & 4,0 & 3,3 & 39,2 & 46,9 & 86,1 & $46 \%$ \\
$2,7-50$ & 26,4 & 2,8 & 2,9 & 42,1 & 53,1 & 95,2 & $44 \%$ \\
$2,7-100$ & 24,2 & 3,3 & 3,6 & 39,1 & 64,6 & 103,7 & $38 \%$ \\
\hline
\end{tabular}

L: Largura; P: Penetração; R: Reforço; Afund: área fundida; AD: área depositada; At: Área Total.

Tabela 4. Parâmetros dimensionais médios e diluição média dos cordões de solda.

\begin{tabular}{ccc}
\hline Parâmetro & Equação & $\mathbf{R}^{2}(\%)$ \\
Largura (L) & $L=0,753 \mathrm{HI}+0,280 \mathrm{HI} \cdot \mathrm{AF}$ & 63,83 \\
Penetração (P) & $P=0,457 \mathrm{HI}$ & 20,93 \\
Reforço (R) & $R=0,724 \mathrm{HI}+0,552 \mathrm{AF}$ & 82,87 \\
Área Depositada (AD) & $A D=0,7789 \mathrm{HI}+0,6031 \mathrm{AF}+0,0811 \mathrm{HI} \cdot \mathrm{AF}$ & 97,65 \\
Área Fundida (Afun) & Afun $=0,793 \mathrm{HI}-0,187 \mathrm{AF}+0,243 \mathrm{HI}-0,337 \mathrm{AF^{2 }}+0,187 \mathrm{HI} \cdot A F$ & 91,42 \\
Área total (At) & $A t=0,8893 \mathrm{HI}+0,4024 \mathrm{AF}+0,1238 \mathrm{HI} \cdot \mathrm{AF}$ & 96,68 \\
Diluição (D) & $D=-0,350 \mathrm{HI}-0,871 \mathrm{AF}+0,171 \mathrm{HI} \cdot \mathrm{AF}$ & 90,71 \\
\hline
\end{tabular}


maior relevância que HI. Esses resultados estão coerentes com as medidas dimensionais como, por exemplo as apresentadas na Figura 5, e mostram que, adicionalmente à energia de soldagem, que já é uma variável bem conhecida e estudada na área de soldagem, a adição de arame frio pode ser usada para afetar os parâmetros dimensionais do cordão de solda, principalmente as áreas depositadas e fundidas, que irão impactar diretamente na diluição, que é extremamente relevante para soldagem de AID.
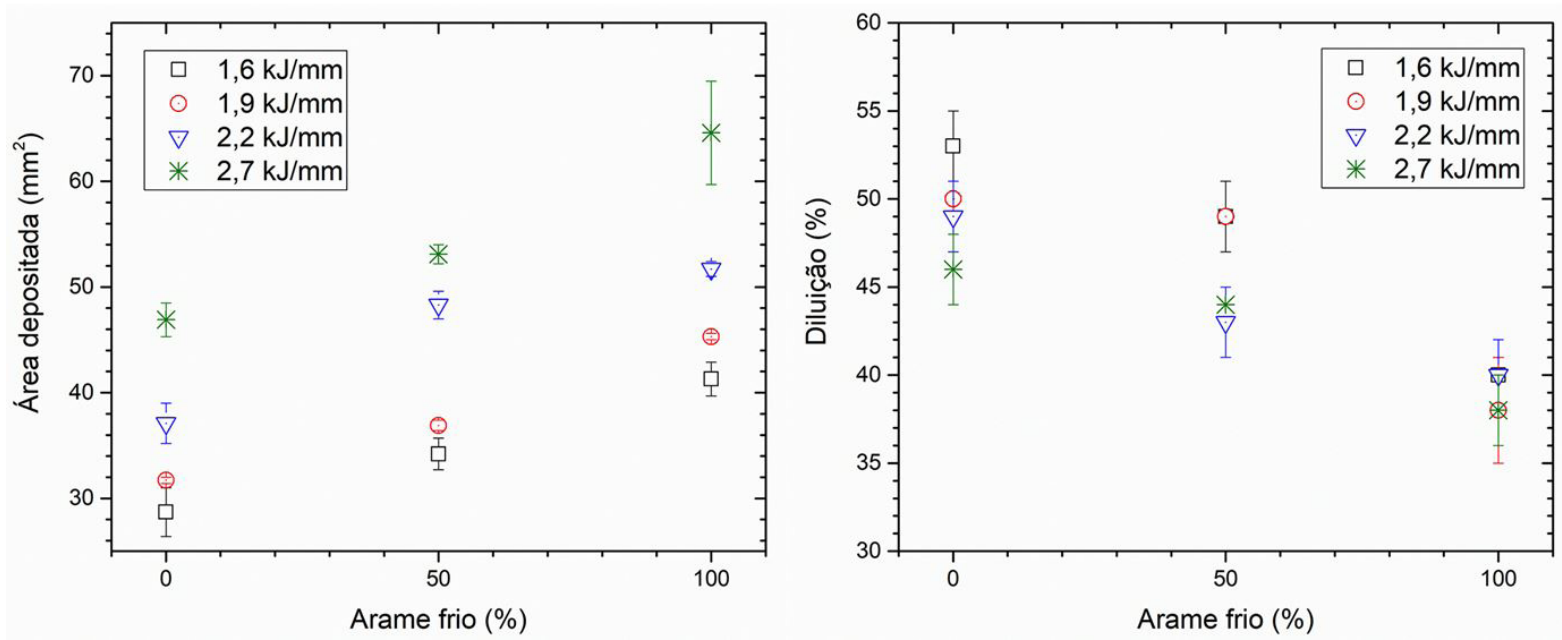

Figura 5. Área depositada (esquerda) e diluição (direita) dos cordões de solda.

A Figura 5 mostra que a diluição reduziu e a área depositada aumentou para maiores AF. O modelamento estatístico apresentado na Figura 6 mostra que quando sem adição de arame frio $(A F=0)$ o aumento da energia de soldagem provocou a redução da diluição, contudo ao se aumentar AF, a $\mathrm{HI}$ passou exercer menor influência sobre esse parâmetro, sendo que para $\mathrm{AF}=100 \%$ a variação da $\mathrm{HI}$ não produziu influência significativa na diluição.

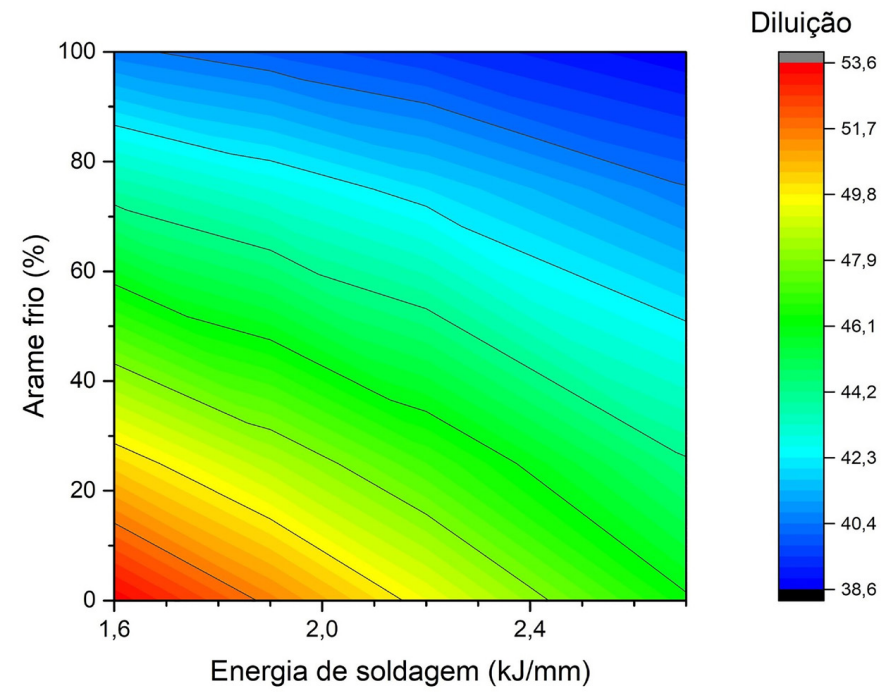

Figura 6. Modelamento estatístico da diluição.

A partir da Figura 7 é possível observar um aumento significativo da TD com o aumento de AF. A regressão linear da TD em função da adição de AF, para as condições de teste usadas, representou um modelo matemático (Equação 3) com $R^{2}$ igual a 0,99922). Ressalta-se que essa equação é válida apenas para a condição de soldagem empregada neste trabalho, ou seja, alterações na corrente de soldagem, velocidade dos arames quentes e DPBC provocariam alterações significativas na TD e demandariam nova coleta de dados. 


$$
T D[\mathrm{~kg} / \mathrm{h}]=4,00 \cdot A F+9,76
$$

A TD aumentou de $19 \%$ de $A F=0 \%$ para $A F=50 \%$ e $18 \%$ deste nível para $A F=100 \%$. Tomando-se os extremos, $\mathrm{AF}=0 \%$ e $\mathrm{AF}=100 \%$, a taxa de deposição aumentou 40,8\%, o que é extremamente relevante para aumento de produtividade, principalmente em situações em que não se pode aumentar a energia de soldagem por limitações metalúrgicas, como no caso dos AID. O aumento da TD pela AF pode ser ainda mais relevante para os AID com maiores teores de elementos de liga, como os super e hiper duplex, uma vez que a máxima energia de soldagem recomendada é menor (de 1,5 kJ. $\mathrm{mm}^{-1}$ ), em função da maior propensão à formação de fases intermetálicas deletérias, como $\chi$ e $\sigma$, associadas à baixa velocidade de resfriamento durante a soldagem [13].
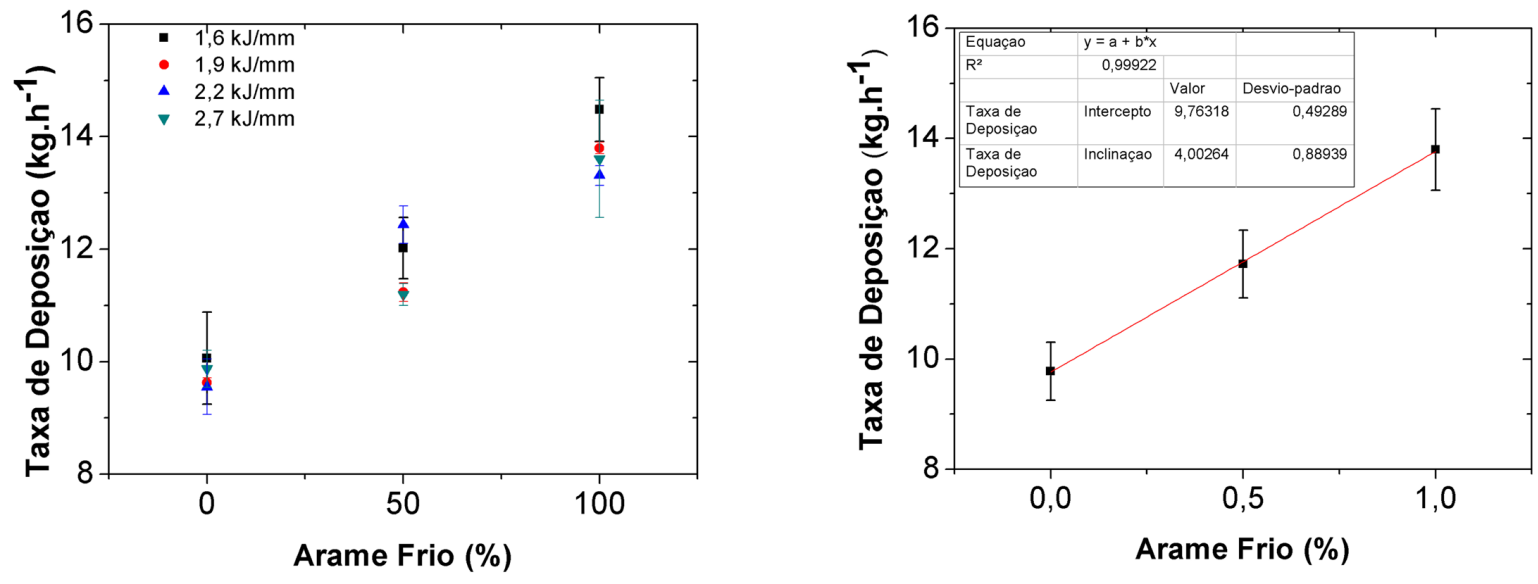

Figura 7. Taxa de deposição das condições testadas (direita) e agrupados por AF (esquerda).

\subsection{Análise química}

A Tabela 5 apresenta a composição química dos cordões de solda. Vale ressaltar que, para os experimentos em questão, a composição do metal de adição é significativamente maior em Ni e Mo em comparação ao metal de base, de forma que a diluição desempenha um importante papel no que diz respeito a composição do MS, e, como consequência, nas suas propriedades metalúrgicas, mecânicas e de resistência à corrosão. Em geral, a adição de AF provocou um aumento do teor de N, Ni e Mo do cordão de solda, o que acarretou no aumento do PREN. Dessa maneira, pode-se afirmar que por meio da adição do AF é possível atuar na composição química da solda, aumentando o seu PREN e possivelmente a resistência à corrosão por pites.

Tabela 5. Parâmetros dimensões e PREN dos cordões de solda. $P R E N=\% C r+3,3 . \% M o+16 . \%$.

\begin{tabular}{|c|c|c|c|c|c|c|c|c|c|c|c|c|}
\hline \multirow{2}{*}{ Condição } & \multicolumn{11}{|c|}{ Concentração dos elementos em peso (\%) } & \multirow{2}{*}{ PREN } \\
\hline & C & Mn & Si & $\mathbf{P}$ & $\mathbf{S}$ & $\mathrm{Cr}$ & $\mathbf{N i}$ & Mo & $\mathrm{Cu}$ & Nb & $\mathbf{N}$ & \\
\hline $1,6-0$ & 0,0106 & 1,2480 & 0,5487 & 0,0201 & 0,0015 & 22,2936 & 6,3919 & 1,7947 & 0,2566 & 0,0097 & 0,1236 & 30,19 \\
\hline $1,6-50$ & 0,0104 & 1,3542 & 0,4868 & 0,0196 & 0,0021 & 22,5149 & 6,4015 & 1,7234 & 0,2517 & 0,0138 & 0,1254 & 30,21 \\
\hline $1,6-100$ & 0,0105 & 1,4110 & 0,4897 & 0,0188 & 0,0016 & 22,5273 & 6,6672 & 1,8931 & 0,2381 & 0,0139 & 0,1306 & 30,86 \\
\hline $1,9-0$ & 0,0118 & 1,2531 & 0,5099 & 0,0202 & 0,0027 & 22,3713 & 6,4464 & 1,7720 & 0,2492 & 0,0138 & 0,1267 & 30,25 \\
\hline $1,9-50$ & 0,0117 & 1,3688 & 0,4997 & 0,0197 & 0,0017 & 22,2846 & 6,3521 & 1,7340 & 0,2671 & 0,0140 & 0,1343 & 30,16 \\
\hline $1,9-100$ & 0,0115 & 1,3880 & 0,4707 & 0,0195 & 0,0021 & 22,3667 & 6,4799 & 1,7879 & 0,2473 & 0,0140 & 0,1284 & 30,32 \\
\hline $2,2-0$ & 0,0111 & 1,2643 & 0,5194 & 0,0203 & 0,0035 & 22,3918 & 6,4941 & 1,7969 & 0,2478 & 0,0140 & 0,1201 & 30,24 \\
\hline $2,2-50$ & 0,0101 & 1,3257 & 0,5128 & 0,0191 & 0,0022 & 22,4934 & 6,7379 & 1,9252 & 0,2337 & 0,0138 & 0,1303 & 30,93 \\
\hline $2,2-100$ & 0,0123 & 1,3720 & 0,4932 & 0,0192 & 0,0022 & 22,4911 & 6,7457 & 1,9470 & 0,2344 & 0,0138 & 0,1288 & 30,98 \\
\hline $2,7-0$ & 0,0107 & 1,2616 & 0,5245 & 0,0201 & 0,0030 & 22,4442 & 6,5669 & 1,8296 & 0,2484 & 0,0137 & 0,1230 & 30,45 \\
\hline $2,7-50$ & 0,0116 & 1,2485 & 0,5213 & 0,0206 & 0,0020 & 22,3304 & 6,6125 & 1,8849 & 0,2459 & 0,0140 & 0,1256 & 30,56 \\
\hline $2,7-100$ & 0,0100 & 1,2457 & 0,6576 & 0,0202 & 0,0024 & 22,2172 & 6,5913 & 1,9541 & 0,2321 & 0,0097 & 0,1248 & 30,66 \\
\hline
\end{tabular}


Avaliação da Energia de Soldagem e da Adição de Arame Frio nas Características Dimensionais, Químicas e Metalúrgicas do Metal de Solda Obtido por Arco Submerso em Aços Inoxidáveis Lean Duplex UNS S32304

O PREN do metal de base utilizado neste trabalho é de $25,07 \%$, assim o PREN do MS é mais elevado que o do metal de base, uma vez que o metal de adição utilizado apresenta maior teor de Mo e $\mathrm{N}$ que o metal de base. Dessa maneira, espera-se que a resistência à corrosão por pites do MS seja maior que a da ZTA e ainda que a mesma aumente com a adição de AF.

\subsection{Microestrutura}

A microestrutura do metal de base (Figura 8) apresenta austenita disposta em forma de lamelas na matriz ferrítica na proporção de $50,5 \pm 1.5 \%$ de austenita e $49,5 \pm 1.5 \%$ de ferrita. Na imagem de $\mathrm{MO}$ as regiões claras são austenita e as escuras são ferrita.
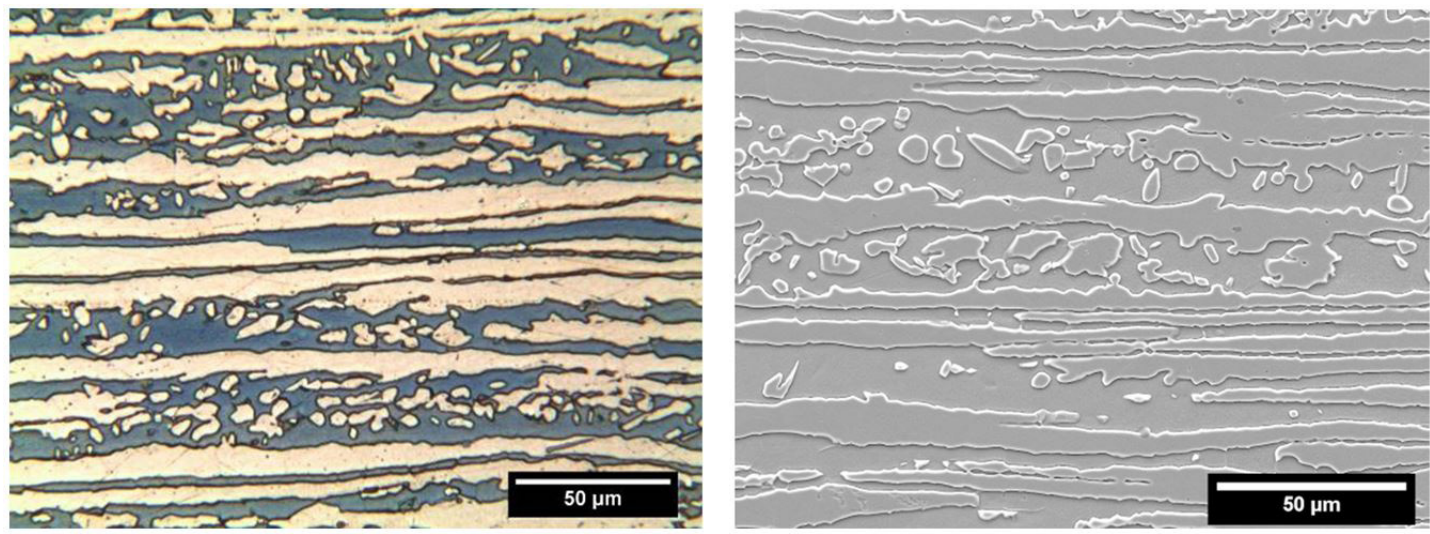

Figura 8. Metal de base UNS S32304: MO (esquerda) e MEV (direita). Ataque: Behara II.

A Figura 9 mostra a microestrutura típica do MS. Foi possível observar a presença de austenita de contorno de grão (GBA), austenita de Widmantätten (WA) e austenita intragranular (IGA) em uma matriz ferrítica no MS de todas as condições. Nesta figura, que apresenta os extremos em termos de adição de AF e HI, fica evidenciada a diferença da fração de austenita entre as duas imagens, sendo que a situação soldada com 1,6 kJ.mm $\mathrm{mm}^{-1}$ e $0 \%$ de AF apresentou visivelmente menor quantidade de austenita que a condição de $2,7 \mathrm{~kJ} \cdot \mathrm{mm}^{-1}$ e $100 \%$ de AF. A maior fração de austenita melhora a tenacidade e diminui a tendência à precipitação de nitreto de cromo, devido à maior solubilidade do nitrogênio na austenita do que na ferrita, o que afeta positivamente a resistência à corrosão por pite [22]. Em contrapartida, um potencial efeito negativo da elevada fração de austenita é o maior risco de trinca a quente durante a solidificação [13].

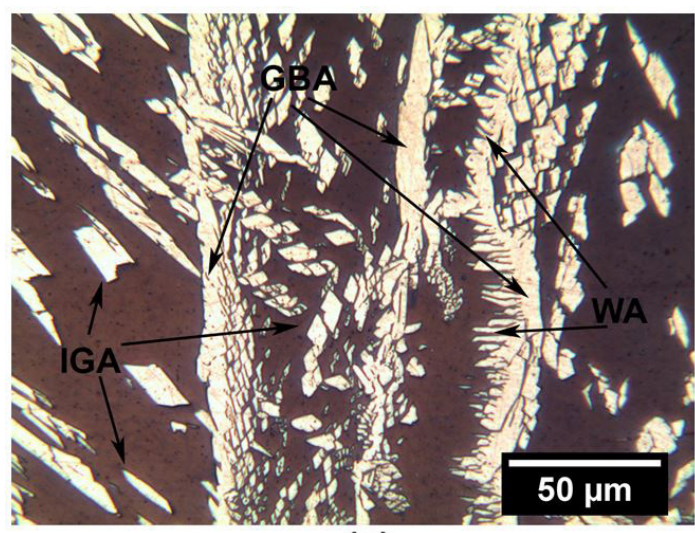

(a)

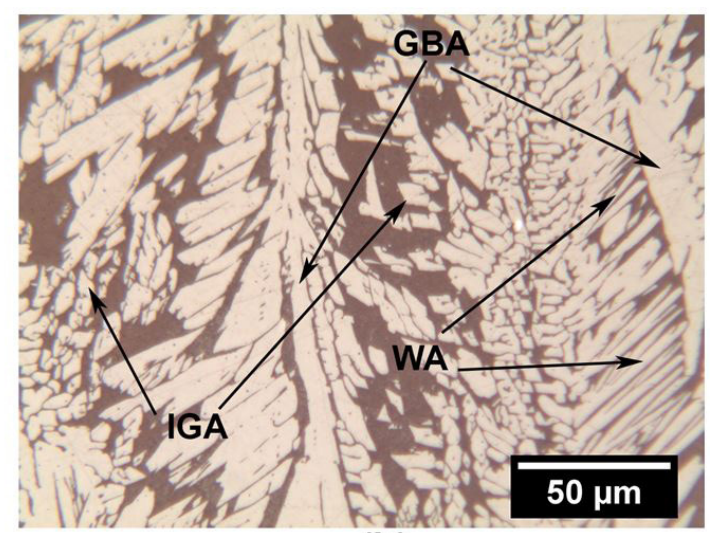

(b)

Figura 9. Microestrutura do MS observada por $\mathrm{MO}$ dos cordões de solda para (a) $\mathrm{HI}=1,6 \mathrm{~kJ} \cdot \mathrm{mm}^{-1}$ e $A F=0$ (1,6-0) e (b) $\mathrm{HI}=2,7 \mathrm{~kJ} \cdot \mathrm{mm}^{-1}$ e $A F=100 \%$ (2,7-100). Sendo GBA: austenita de contorno de grão, WA: austenita de Widmantätten e IGA: austenita intragranular. Ataque: Behara II. 
Por meio do MEV, foi observado a presença de inclusões globulares tanto na austenita quanto na ferrita do MS, contudo não se observou a presença de precipitados como fase $\sigma, \chi$, nitreto de cromo, dentre outras. A Figura 10 apresenta a morfologia das inclusões encontradas no MS da amostra 1,6-100, a qual representa o aspecto esférico, típico dessas inclusões em todas as amostras, enquanto a Tabela 6 apresenta a composição química da mesma obtida por EDS, que mostra que tal inclusão é rica em O e Si e elementos como $\mathrm{Mn}$ e $\mathrm{Cr}$, sendo possível, portanto, caracterizá-las como óxidos e/ou silicatos desses elementos. Tais inclusões não metálicas são originadas durante a solidificação do MS e estão presentes em maiores quantidades em processos de soldagem com geração de escória, como o SAW [23]. Cardoso [24] observou inclusões semelhantes em morfologia para soldas de composição químicas semelhantes à deste trabalho obtidas pelos processos SMAW, GMAW e FCAW.
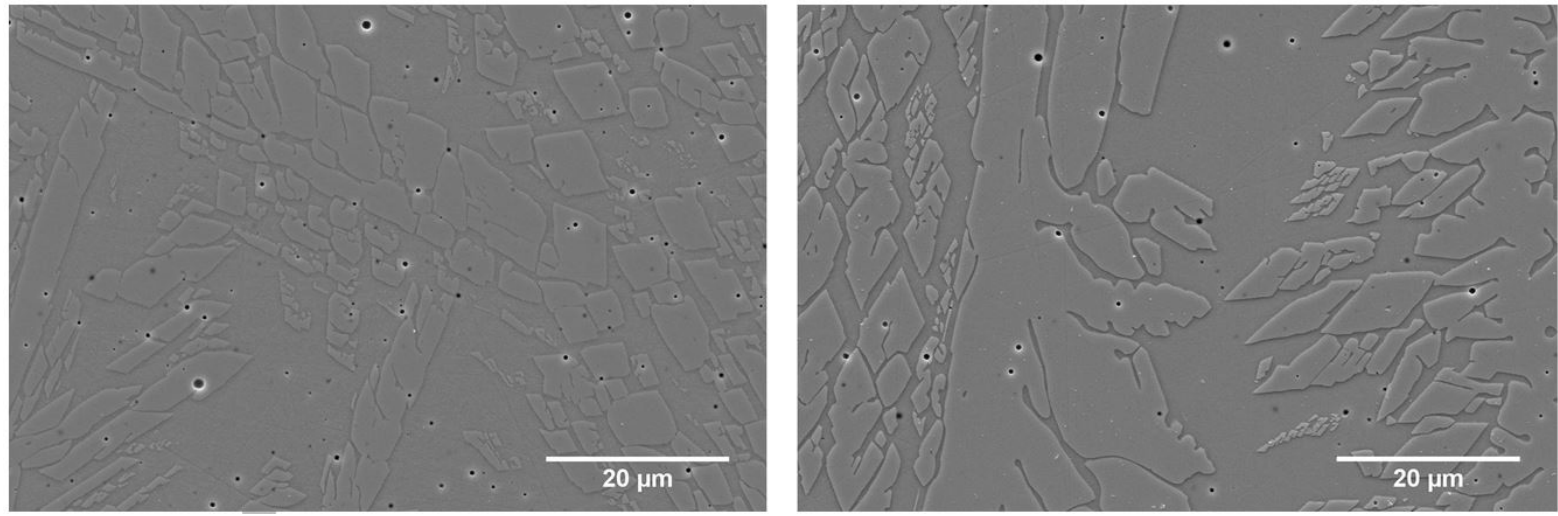

Figura 10. MEV do MS da amostra 1,6-100 (esquerda) e 2,7-100 (direita). Ataque: Behara II.

Tabela 6. Composição química em peso de uma inclusão de $6 \mu \mathrm{m}$ de diâmetro aproximado da amostra 1,6-100. MEV/EDS.

\begin{tabular}{ccccccccccccc}
\hline Elemento & $\mathbf{O}$ & $\mathbf{N a}$ & $\mathbf{M g}$ & $\mathbf{A l}$ & $\mathbf{S i}$ & $\mathbf{M o}$ & $\mathbf{K}$ & $\mathbf{C a}$ & $\mathbf{C r}$ & $\mathbf{M n}$ & $\mathbf{F e}$ \\
$\mathrm{pp}(\%)$ & 38,63 & 8,77 & 0,32 & 18,67 & 19,24 & 0,16 & 1,85 & 1,01 & 1,72 & 7,3 & 2,34 \\
\hline
\end{tabular}

A Figura 11 apresenta a fração de austenita e de inclusões não metálicas no MS. A fração de austenita no MS aumentou com o aumento de $\mathrm{AF}$ e da $\mathrm{HI}$, sendo a primeira relacionada a composição química mais rica em $\mathrm{Ni}$ e $\mathrm{N}$ pela menor diluição, enquanto a segunda associada tanto à menor velocidade de resfriamento, o que favorece a decomposição da ferrita em austenita, quanto à menor diluição. O modelo estatístico mostrou que, apesar da influência de AF ser maior que de HI, a diluição também reduz quando HI aumenta. Vale ressaltar que o balanço de fases é um dos fatores mais importantes durante a soldagem de AID [13], sendo que a presença excessiva de ferrita

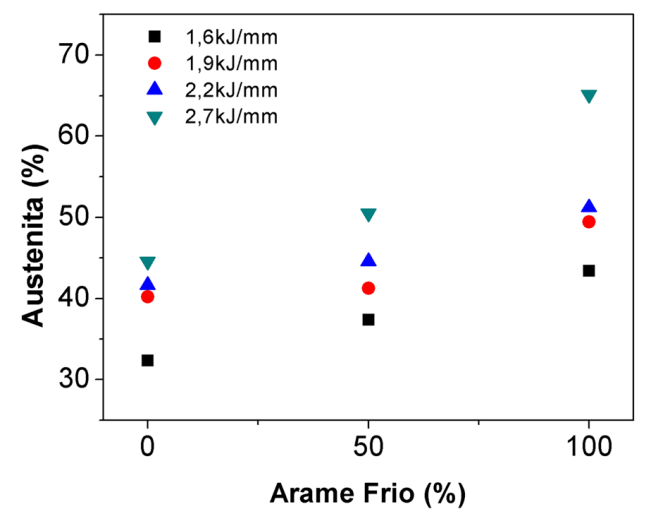

(a)

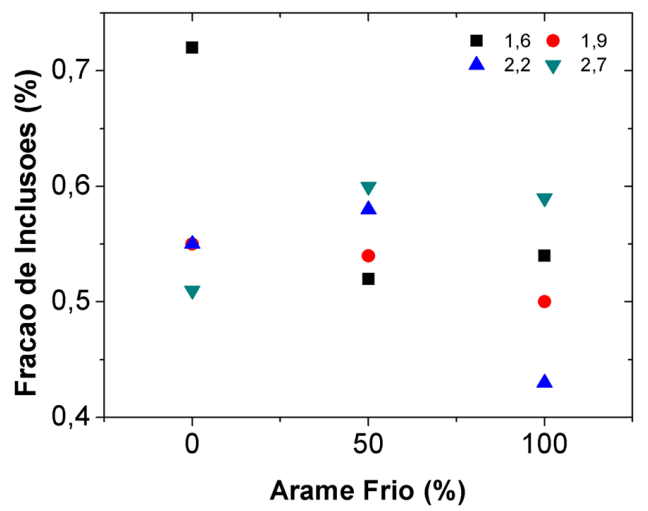

(b)

Figura 11. Fração de austenita (a) e fração de inclusões não metálicas (b) no MS. 
Avaliação da Energia de Soldagem e da Adição de Arame Frio nas Características Dimensionais, Químicas e Metalúrgicas do Metal de Solda Obtido por Arco Submerso em Aços Inoxidáveis Lean Duplex UNS S32304

pode levar à formação de nitretos de cromo, comprometendo a ductilidade, tenacidade e resistência à corrosão [9]. Neste caso, durante o resfriamento rápido da soldagem, como não há tempo suficiente para difusão do nitrogênio para a austenita e sua solubilidade na ferrita é muito baixa, o limite de solubilidade de $\mathrm{N}$ na ferrita é excedido e ocorrem as reações de precipitação de nitreto de cromo [9], que aparecem predominantemente como $\mathrm{Cr}_{2} \mathrm{~N}[23,25]$.

As medidas de fração de inclusões (Figura 11 (b)) apresentaram uma dispersão considerável não possibilitando uma análise confiável dos efeitos da HI e de AF em relação à essa variável, o que era esperado considerando que a quantidade de inclusões foi relativamente pequena. Não se observou uma relação clara entre a quantidade de inclusões e as condições de ensaio. Também não foi encontrada na literatura qualquer consideração com relação a este aspecto.

\section{Conclusões}

Através da adição de AF foi possível aumentar a taxa de deposição sem o aumento da $\mathrm{HI}$, de forma que esse processo representa uma efetiva alternativa para aumento de produtividade na soldagem de aços inoxidáveis "lean" duplex UNS S32304, que possuem faixa restrita de utilização de energia de soldagem.

Além disso, a adição de $\mathrm{AF}$ e a $\mathrm{HI}$ afetaram a geometria do cordão de solda. O principal impacto resultante foi uma redução significativa da diluição com o aumento da adição de AF, influenciando, portanto, a composição química do MS para uma mesma energia de soldagem. Uma vez que o material de adição é mais ligado ao Ni, Mo e $\mathrm{N}$ que o material de base, as soldas com maiores AF apresentaram-se mais ricas nesses elementos. Assim, as soldas com maiores AF apresentaram maiores PREN e devem possuir maior resistência à corrosão localizada. Em relação à microestrutura, o aumento de AF provocou o aumento da fração de austenita no MS. $\mathrm{O}$ aumento da HI também aumentou a fração de austenita do MS, como esperado. Foram encontradas inclusões não metálicas caracterizadas como óxidos e/ou silicatos, entretanto não foi observada uma tendência clara da quantidade destas inclusões em relação as variáveis estudadas.

Assim, com a adição de AF foi possível aumentar a produtividade e influenciar as propriedades do MS, de forma que é possível afirmar que a adição de AF possivelmente irá provocar um efeito positivo na tenacidade (pela maior fração de austenita e menor de inclusões não metálicas) e na corrosão localizada (pelo maior PREN). Em contrapartida, um maior cuidado deverá ser tomado na soldagem em relação à trinca a quente, a qual é favorecida pelo aumento da fração de austenita. Trabalhos adicionais serão realizados para confirmar esses pontos.

\section{Agradecimentos}

A Aperam South América pelo fornecimento do aço e suporte, a ESAB Industria e Comércio LTDA pelo fornecimento dos consumíveis e soldagem e a UFMG e CDTN pelo fornecimento da infraestrutura para execução das análises. Ao CAPES-PROEX, CNPq e FAPEMIG pelo apoio financeiro.

\section{Referências}

[1] Cardoso R Jr, Bracarense AQ, Campos FR, Souza CS, Silveira DM, Lins VFC. Avaliação da soldagem multipasse de chapas espessas de aços inoxidáveis Lean Duplex UNS S32304 soldadas pelos processos SMAW, GMAW e FCAW - parte 1: propriedades mecânicas. Soldagem e Inspeção. 2012;17(4):306-316. http:// dx.doi.org/10.1590/S0104-92242012000400005.

[2] Alvarez-Armas I. Duplex stainless steels: brief history and some recent alloys. Recent Patents on Mechanical Engineering. 2008;2008(1):51-57. http://dx.doi.org/10.2174/22127976108 01010051.

[3] Olsson J, Liljas M. 60 years of DSS applications. In: Proceedings of the NACE Corrosion 94 Conference; 1994 February-March 27-04; Baltimore. Yucca Mountain, NV: Center for Nuclear Waste Regulatory Analyses - CNWRA; 1994. p. 395.

[4] Pohl M. The Ferrite/Austenite ratio of duplex stainless steels. Zeitschrift fur Metallkunde. 1995;86(2):97-102.

[5] Rebello JMA, Mattos OR, Nunes FC, Matos TF, Salustiano AZ, Santos NB, et al. Aços duplex e superduplex: estado da arte. Rio de Janeiro: COOPETEC Fundação; 2006.

[6] Olsson COA. The influence of nitrogen and molybdenum on passive films formed on the austen oferritic stainless steel 2205 studied by AES and XPS. Corrosion Science. 1995;37(3):467-479. http://dx.doi.org/10.1016/0010-938X(94)00148-Y.

[7] Westin EM. Microstructure and properties of welds in the lean duplex stainless steel LDX $2101^{\circledR}$ [doctor thesis]. Stockholm, Sweden: Royal Institute of Technology; 2010.

[8] Alvarez-Armas I and Degallaix-Moreuil S. Duplex stainless steel. Hoboken: John Wiley \& Sons, Inc.; 2009. http://dx.doi. org/10.1002/9781118557990.

[9] Lippold JC and Kotecki DJ. Welding metallurgy and weldability of stainless steels. Hoboken: John Wiley \& Sons, Inc.; 2005. $376 \mathrm{p}$. 
[10] Jiang Y, Tan H, Wang Z, Hong J, Jiang L, Li J. Influence of Creq/ Nieq on pitting corrosion resistance and mechanical properties of UNS 32304 duplex stainless steel welded joints. Corrosion Science. 2013;70:252-259. http://dx.doi.org/10.1016/j. corsci.2013.01.037.

[11] Chehuan T, Dreilich V, Assis KS, Sousa FVV, Mattos OR. Influence of multipass pulsed gas metal arc welding on corrosion behaviour of a duplex stainless steel. Corrosion Science. 2014;86:268-274. http://dx.doi.org/10.1016/j.corsci.2014.06.004.

[12] Kim HJ, Jeon SH, Kim ST, Lee IS, Park YS, Kim KT, et al. Investigation of the sensitization and intergranular corrosion of tube-to-tubesheet welds of hyper duplex stainless steel using an electrochemical reactivation method. Corrosion Science. 2014;87:60-70. http://dx.doi.org/10.1016/j.corsci.2014.06.005.

[13] Kalsson L. Welding duplex stainless steels - a review of current recommendations. Welding in the World. 2012;56(5-6):65-76.

[14] Ramakrishnan M, Muthupandi V. Application of submerged arc welding technology with cold wire addition for drum shell long seam butt welds of pressure vessel components. International Journal of Advanced Manufacturing Technology. 2012;65(58):1-12. https://doi.org/10.1007/s00170-012-4230-0.

[15] Xiang T, Li H, Wei HL, Gao Y. Effects of filling status of cold wire on the welding process stability in twin-arc integrated cold wire hybrid welding. International Journal of Advanced Manufacturing Technology. 2016;83(9-12):1583-1593. http:// dx.doi.org/10.1007/s00170-015-7686-x.

[16] Mohammadijoo M, Collins L, Henein H, Ivey DG. Evaluation of cold wire addition effect on heat input and productivity of tandem submerged arc welding for low-carbon microalloyed steels. International Journal of Advanced Manufacturing Technology. 2017;92(1-4):1-13.

[17] Castro MM. Influência da adição de arame frio na morfologia do cordão na soldagem ao arco submerso com dois arames
TWIN [dissertação de mestrado]. Belo Horizonte: Universidade Federal de Minas Gerais; 2016.

[18] American Society of Mechanical Engineers. Section II - materials part C - specifications for welding rods electrodes and filler metals. New York: ASME; 2017.

[19] American Society for Testing And Materials. A240 / A240M17: standard specification for chromium and chromium-nickel stainless steel plate, sheet, and strip for pressure vessels and for general applications. West Conshohocken: ASTM; 2017.

[20] The Materials Information Society. Metallography and microstructures. Vol. 9. Ohio: ASM International; 2004. (ASM Handbook)

[21] Quinino RC, Reis EA, Bessegato LF. O coeficiente de determinação R2 como instrumento didático para avaliar a utilidade de um modelo de regressão linear múltipla. Belo Horizonte: UFMG; 1991.

[22] Liou HY, Hsieh RI, Tsai WT. Microstructure and Pitting Corrosion in Simulated Heat-affected Zones of Duplex Stainless Steels. Materials Chemistry and Physics. 2002;74(1):33-42. http:// dx.doi.org/10.1016/S0254-0584(01)00409-6.

[23] Brand SD, Ramirez AJ. Precipitation of intermatallic phases in the HAZ of multipass welding of duplex and superduplex stainless steels. In: Proceedings of the 5th World Conference Duplex Stainless Steel 97; Outubro 1997; Maastricht, Holanda. Zutphen: $\mathrm{KCl}$ Publishing; 1997. pp. 405-411.

[24] Cordoso R Jr. Avaliação da soldagem multipasse de chapas espessas do aço inodável lean duplex UNS S32304 soldadas pelos processos SMAW, GMAW E FCAW [dissertação de mestrado]. Belo Horizonte: Universidade Federal de Minas Gerais; 2012.

[25] Brand SD, Lippold JC. The corrosion resistance of simulated multipass welds of duplex stainless steels. In: Proceedings of the 5th World Conference Duplex Stainless Steel 97; Outubro 1997; Maastricht, Holanda. Zutphen: KCI Publishing; 1997. pp. 411-418. 